\title{
Impact of BRCA1/2 gene mutations on survival of patients with pancreatic cancer: A case-series analysis
}

\author{
Ernesto Barzola Navarro', Emilio Vicente López ${ }^{1}$, Yolanda Quijano ${ }^{1}$, Riccardo Caruso ${ }^{1}$, Valentina Ferri ${ }^{1}$, \\ Hipolito Durand ${ }^{1}$, Isabel Fabra Cabrera', Eduardo Diaz Reques ${ }^{1}$, Benedetto lelpo ${ }^{1}$, \\ Anastasiia Yuriyivna Glagolieva ${ }^{2}$, and Carlos Plaza ${ }^{3}$
}

\author{
${ }^{1}$ Department of General and Digestive Surgery, Hospital Universitario Madrid Sanchinarro, Madrid, Spain, \\ ${ }^{2}$ Department of Surgery and General Surgery, Shupyk National Medical Academy of Postgraduate Education, Kyiv, \\ Ukraine, ${ }^{3}$ Department of Pathology, Hospital Universitario Madrid Sanchinarro, Madrid, Spain
}

\begin{abstract}
BRCA gene mutations are found in up to $10 \%$ of pancreatic adenocarcinoma cases. We present a description of 4 cases along with a review of the current literature regarding pathogenesis, target treatment, response and survival rates in these types of malignancies. We describe four cases of pancreatic adenocarcinoma, in three of which the BRCA2 mutation was identified, in one - BRCA1 gene alteration. Two patients underwent surgery following the neoadjuvant treatment with Folfirinox and radiotherapy; in the first case, a distal pancreatectomy with splenectomy was performed and in the second one - the Whipple's procedure. In both cases, a complete pathological response was reported. Other 2 patients were treated with Folfirinox after BRCA mutation identification and acceptable life expectancy was obtained. The association of pathologic complete response (PCR) with lower rates of local recurrence and better survival in patients with various types of adenocarcinomas is well known. Identification of such patients carrying BRCA mutations could provide an application of better personalized treatment. In some patients with pancreatic cancer, especially when there is clinical or demographic reason to suspect a genetic predisposition, a confirmation of the presence of BRCA mutations could provide an opportunity to use target treatment with beneficial outcomes regarding survival. (Ann Hepatobiliary Pancreat Surg 2019;23:200-205)
\end{abstract}

Key Words: Pancreatic adenocarcinoma; BRCA mutations; Cisplatin; Folfirinox; Pathologic complete response

\section{INTRODUCTION}

The median survival of pancreatic ductal cancer today comprises less than 1 year for advanced stages of the disease, and even when early diagnosed, the 5-year survival for the above malignancy reaches only $7 \%{ }^{1}$

Around $10 \%$ of the pancreatic ductal adenocarcinoma (PDAC) are associated with hereditary predisposition. ${ }^{2}$ BRCA2 mutations increase the risk of breast cancer, prostate cancer, pancreatic cancer, and melanoma and stomach cancer. ${ }^{3}$ It is estimated that in BRCA2 carriers, the cumulative risk of all cancers is $32 \%$ for men by the age of 70 years, and $90 \%$ for women. ${ }^{4}$ The estimated lifetime risk of pancreatic cancer for individuals with a BRCA2 mutation is $4.9 \%{ }^{5}$ The cases of familial pancreatic cancer
(FPC) have also been reported. ${ }^{6}$ Researchers have documented germinal gene mutations which are known to increase the risk of PDAC.

The effect of BRCA mutations and response to DNA crosslinking agents in PDA was evaluated and there are authors who reported that BRCA-associated PDA in patients receiving a platinum agent as first-line metastatic therapy demonstrated a partial or complete radiographic response. $^{7}$

Lowery et al. ${ }^{8}$ have been studying the effect of BRCA mutations and response to DNA crosslinking agents in PDAC. It was reported that 5/6 BRCA-associated PDAC patients who received a platinum agent as first-line metastatic therapy demonstrated a partial or complete radiographic response (Table 1). This favorable response of

Received: September 3, 2018; Revised: December 10, 2018; Accepted: December 14, 2018

Corresponding author: Ernesto Barzola Navarro

Department of General and Digestive Surgery, Hospital Universitario Madrid Sanchinarro, Calle de Oña, 10, Madrid 28050, Spain Tel: +34650409788, Fax: +34650409788, E-mail: drbarzola@gmail.com

Copyright (C) 2019 by The Korean Association of Hepato-Biliary-Pancreatic Surgery

This is an Open Access article distributed under the terms of the Creative Commons Attribution Non-Commercial License (http://creativecommons.org/ icenses/by-nc/4.0) which permits unrestricted non-commercial use, distribution, and reproduction in any medium, provided the original work is properly cited. Annals of Hepato-Biliary-Pancreatic Surgery • pISSN: 2508-5778 - eISSN: 2508-5859 
Table 1. Patients with BRCA1/2 mutations and pathological or radiological response after treatment reported in literature

\begin{tabular}{|c|c|c|c|c|c|}
\hline Authors & Year & Patients & BRCA & CXRT & Response reported \\
\hline Sonnenblick et al. ${ }^{9}$ & 2011 & 1 & BRCA 2 & Cisplatinum & Radiological \\
\hline Lowery et al. ${ }^{8}$ & 2011 & 15 & $\begin{array}{l}\mathrm{BRAC} 2=11 \\
\mathrm{BRAC} 1=4\end{array}$ & Cisplatinum & Radiological \\
\hline Golan et al. ${ }^{20}$ & 2017 & 71 & $\begin{array}{l}\mathrm{BRAC} 2=49 \\
\mathrm{BRAC} 1=21 \\
\mathrm{BRCA} 1 / 2=1\end{array}$ & Gem-Cis & NR \\
\hline Gostimir et al. ${ }^{23}$ & 2016 & 1 & BRCA 2 & Folfirinox & Pathological \\
\hline Vyas et $\mathrm{al}^{22}$ & 2015 & 10 & BRCA2 & Gem-Cis/Oxa/Iri & Radiological \\
\hline Zhao et al. ${ }^{21}$ & 2012 & $11 / 442$ & NR & Gem-Cis-XRT & Pathological \\
\hline
\end{tabular}

NR, not reported; Cis, cisplatinum; Gem, Gemcitabina; CXRT, Chemoradioterapy

Table 2. Summary of clinical information, neoadjuvant therapy schemes and outcomes

\begin{tabular}{ccccccccc}
\hline Case & Age & Stage & Localization & Size mm & CRT & BRCA & Demo & OS (mo) \\
\hline 1 & 63 & uT3N1 & Neck & $37 \times 21$ & FOX & 2 & Yes & $9 \mathrm{~m}$ \\
2 & 46 & uT3N1 & Head & $27 \times 55$ & FOX & 2 & Yes & $15 \mathrm{~m}$ \\
3 & 60 & uT3N1 & Head & $37 \times 20$ & GEM+P-FOX & 2 & No & $36 \mathrm{~m}$ \\
4 & 56 & uT4N1 & Body & $30 \times 40$ & GEM+P-FOX & 1 & Yes & $14 \mathrm{~m}$ \\
\hline
\end{tabular}

CXRT, chemoradiotherapy treatment (50.4 G/y); FOX, folfirinox; GEM+P-, Gemcitabine+Paclitaxel; OS, over survival

BRCA-associated PDAC to DNA crosslinking agents is also supported by a case report by Sonnenblick. ${ }^{9}$ A survival advantage with platinum-containing regimens was reported also for patients with PDAC and a family history of any malignancy, in whom the status of BRCA mutation carrier was not specified. ${ }^{10}$

Mutations in BRCA 2 are considered to be the most common accounting for up to $17 \%$ of FPC. ${ }^{11}$ Hereby, we discuss four cases of pancreatic cancer and the potential role of BRCA mutations regarding therapeutic implications, complete pathological response and survival benefits (Table 2).

\section{CASE}

\section{Case 1}

A 63-year-old woman was referred to emergency department with sudden epigastria pain reporting that pain had started 1 month ago. Abdominal ultrasound (US) revealed a mass in pancreas. On CT scans, a lesion of 30 $\mathrm{mm}$ in the pancreatic neck was found. PET-CT showed an increased uptake in the head of the pancreas (SUV: 7.45) (Fig. 1). Eco-endoscopy identified a solid mass uT3N1, and the biopsy results were compatible with adenocar- cinoma. From the family history, mother of the patient had endometrial cancer, sister suffered from breast cancer, and aunt also had breast cancer, all of them were BRCApositive. The patient underwent the BRCA test, and the BRCA2 mutation was identified. Neoadjuvant chemotherapy with FOLFIRINOX was started with excellent tolerance after 6 cycles and subsequent radiotherapy was applied. PET-MRI visualization revealed the absence of pathological uptake and ruled out any metastatic lesions (Fig. 1). A standard distal pancreatectomy with splenectomy was performed. The pathology results showed complete response to therapy with no signs of malignancy. Currently the patient (Fig. 2) is asymptomatic with no evidence of recurrence at 9-month follow-up.

\section{Case 2}

A 46-year-old female presented with jaundice and bilirubin level up to $6.1 \mathrm{mg} / \mathrm{dl}$. Her sister had oncological history of breast cancer. Thoracic-abdominal-pelvic CT was performed and ultrasound-guided fine-needle biopsy was suggestive for adenocarcinoma uT3N1 in the head of pancreas. The patient underwent the placement of biliary metallic stent and received 7 cycles of neoadjuvant therapy with folfirinox and subsequent radiotherapy. The Whipple 

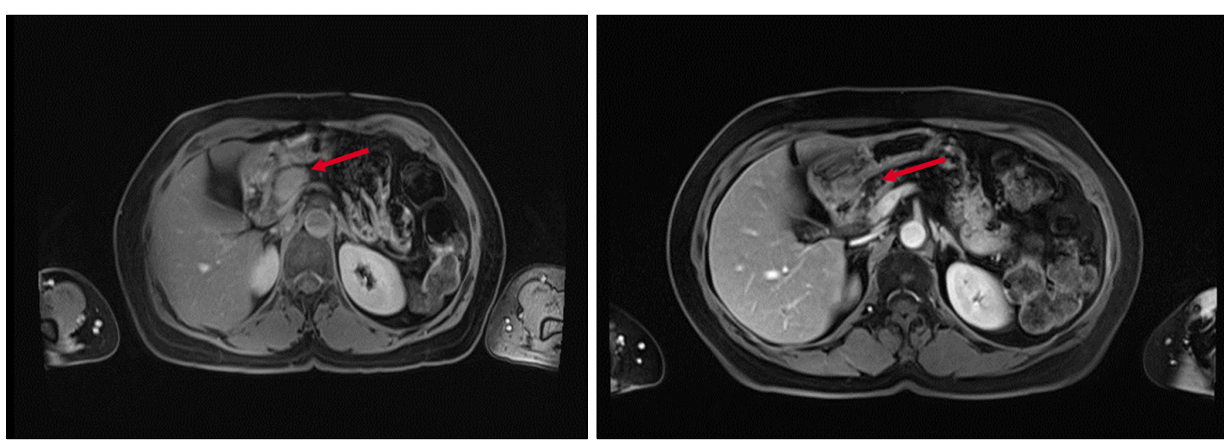

Fig. 1. Axial magnetic resonance imaging (MRI) images before (right) and after (left) the therapy in the neck of pancreas (red arrow), marked tumor shrinkage after neoadjuvant chemoradiation is indicated with the arrow on the second MRI-scan.

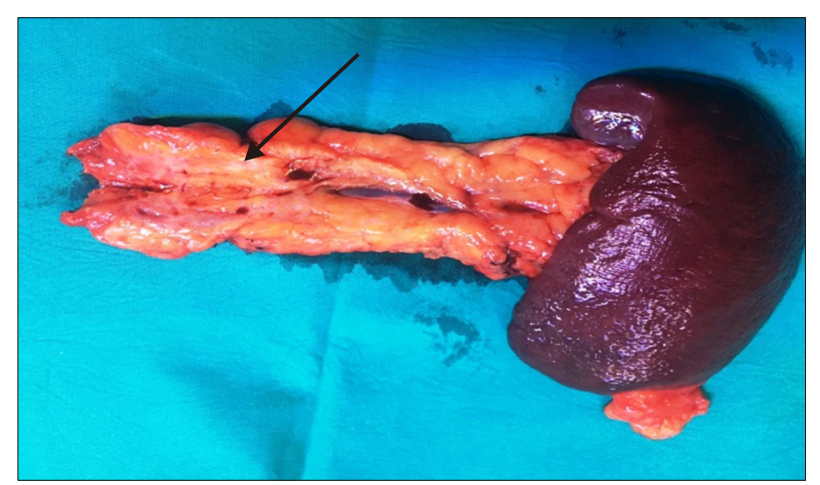

Fig. 2. Intraoperative findings (fibrosis) in the specimen.

procedure was performed and pathological examination revealed complete pathologic response (Fig. 3). The adjuvant therapy with Gemcitabine was prescribed. For now, the patient remains asymptomatic and with no signs of tumor recurrence after 15 months of follow-up.

\section{Case 3}

A 56-year-old female presented with a history of breast cancer treated with surgery and chemoradiotherapy 9 years ago. The previous year she started experiencing abdominal pain and the thoracic-abdominal CT scan revealed a solid mass in the pancreas $30 \times 40 \mathrm{~mm}$ in size. Ultrasoundguided fine-needle biopsy confirmed adenocarcinoma uT4N1 in the pancreatic neck with vascular infiltration. The patient received neoadjuvant therapy with Gemcitabine and paclitaxel with following radiotherapy. Whipple procedure was performed and the histopathological study revealed pancreatic adenocarcinoma pT3N0. The adjuvant therapy with Gemcitabine was prescribed. The follow up examination identified liver metastases; the BRCA test confirmed the presence of BRAC1 mutation. Finally, the patient received chemotherapy with Folfirinox with good response and no signs of metastatic disease progression.

\section{Case 4}

A 60-year-old male presented with jaundice and abdominal pain having elevated liver function indices. Abdominal CT scan demonstrated a lesion in the head of the pancreas measuring $37 \mathrm{~mm}$ with common bile duct and hepatic duct dilatation and another lesion in liver compatible with metastases. After echoendoscopy, the findings were staged as uT3N1M1 tumor. The biopsy confirmed pancreatic adenocarcinoma. The patient underwent neoadjuvant treatment with gemcitabine and abraxane, after the follow-up examination, this treatment was changed due to suspected progression of the disease regarding the elevation of CA19-9 serum marker; finally, in 2014, the FOLFIRINOX and radiotherapy were prescribed. The laboratory studies revealed BRCA2 mutation. Until 2018, a response to therapy is preserved with no evidence of metastatic disease.

\section{DISCUSSION}

PDAC represents a challenging issue in the era of modern oncology. Despite the progress in the research and treatment efficacy of the above pathology, the incidence of all types of pancreatic cancer $(85 \%$ of which are adenocarcinomas) ranges from 1 to 10 cases per 100,000 people. ${ }^{5}$ This rate is generally higher in developed countries and among males remaining at the same level over the past 30 years relative to the incidence of other common solid tumors. ${ }^{5,12}$ It is the eighth leading cause of death from cancer among men and the ninth in women throughout the world. ${ }^{12}$

Features of pancreatic adenocarcinoma include a very high rate of activating mutations. Germline mutations in the tumor suppressor genes of breast cancer, namely, BRCA1 and BRCA2 have been proven to portend a dras- 

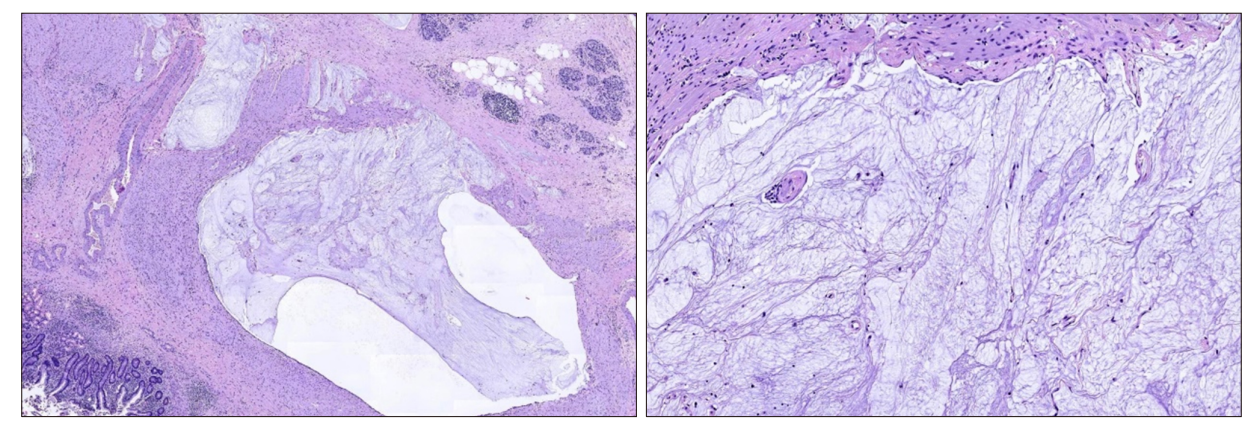

Fig. 3. Microphotograph images of complete tumor response with absence of tumor cells that are replaced by extensive quantities of mucus and reparative mesenchymal cells $(\times 2)$.

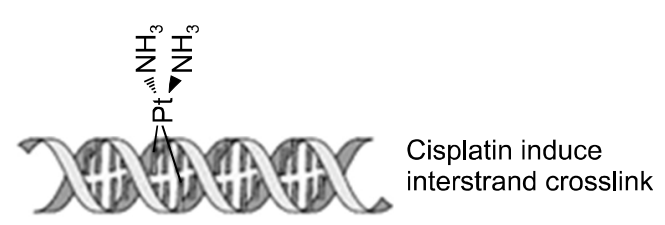

Replication leads to DSB

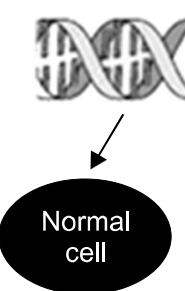

DSB repaired $\downarrow$

Cell survival

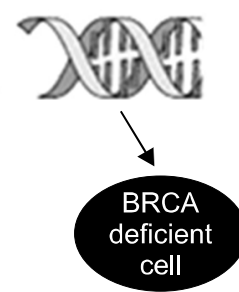

Deficient HR,

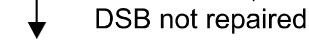

Cell death

Fig. 4. BRCA deficient cells are more sensitive to DNA crosslinking agents such as cisplatin. $\mathrm{hR}$, homologous recombination; dsB, double strand breaks [from Sonnenblick et al., 2011].

tically increased lifetime risk of breast and ovarian cancers in the alteration carriers. A number of studies has shown that the third most common cancer associated with these mutations is pancreatic cancer. ${ }^{13}$

In a prospective study, a statistically significant 2.4 -fold increase in pancreatic cancer incidence was found in female $B R C A$ mutation carriers compared with the general population. ${ }^{14}$ Women with a $B R C A$ mutation who were over the age of 50 had an annual risk of developing pancreatic cancer of 0.04 percent. If these female $B R C A$ mutation carriers had a first-degree relative with pancreatic cancer, the annual risk for developing pancreatic cancer was 1 percent. $^{5}$

BRCA1 and BRCA2 are tumor suppressor genes that are inherited in an autosomal dominant fashion with incomplete penetrance. The loss of function of the tumor suppressor genes is essential in the cascade of genetic changes that causes a failed control of the cell growth and differentiation and drives tumorigenesis. Both BRCA proteins are engaged in transcriptional regulation of gene expression as well as the recognition or repair of DNA damage, particularly double-strand breaks. In patients with sporadic pancreatic cancer, BRCA1/2 are mutated in the most advanced pancreatic intraepithelial neoplasia lesions, whereas a germline mutation in either of the genes represents the earliest risk factor in many types of FPC. ${ }^{4,5,12}$

While the BRCA1 plays a pivotal role in the initiation of the process of DNA repair, the BRCA2 directly participates in the reparation apparatus creating a complex with the RAD51 (an essential protein for DNA repair for homologous recombination) (Fig. 4) in the foci of DNA breaks. $^{8}$

Clinical characteristics associated with the $B R C A$ mutations are following: women who develop breast cancer at age of 40 or younger are at increased risk for positive results of BRCA mutation testing, particularly if they originate from Ashkenazi Jewish ethnicity; ${ }^{15}$ triple-negative breast cancers; ${ }^{16}$ up to 14 percent of men with breast cancer have a BRCA2 mutation. ${ }^{17}$ Among women with ovarian cancer, regardless of family history, about 15 percent are attributable to $B R C A$ mutations. ${ }^{18}$

Neodjuvant chemoradiotherapy improves the survival for resectable PADC and prognosis for loco-regional metastatic disease. Recently, the multidrug chemotherapy regimens such as a combination of fluorouracil, irinotecan, oxaliplatin, and leucovorin (FOLFIRINOX) and gemcitabine plus albumin bound to paclitaxel particles (nab-paclitaxel) have gained popularity in the preoperative and postoperative settings based on their efficiency in patients with metastatic disease. ${ }^{11,19}$ Platinum agents exert their antineoplastic activity by causing DNA crosslinking. Addition of DNA cross-linking agent such as cisplatin to stand- 
ard gemcitabine chemotherapy or FOLFIRINOX regimen should be considered in BRCA mutation carriers. ${ }^{13}$

Platinum analogues are DNA cross-linking agents, which kill tumor cells by creating DNA lesions during S-phase, probably inhibiting DNA replication. ${ }^{9}$ Regarding the role of BRCA in DNA repair, it is speculated that mutations in BRCA1 and BRCA2 result, on the one hand, in the increased cancer incidence due to defective homologous recombination, which leads to genome instability, but, on the other hand, these cancers are more sensitive to DNA cross-linking agents such as cisplatin or oxaliplatin.

We report 2 cases of pancreatic adenocarcinoma associated with BRCA2 mutations where a good response to the treatment was observed. Similar favorable response of BRCA-associated PDA to DNA crosslinking agents was also reported in a series by Sonnenblick et al. ${ }^{9}$ (Table 1). ${ }^{8,9,20-23}$ A survival advantage under application of platinum-containing regimens was also reported for patients with PDA and a family history of any malignancy, in whom the status of BRCA mutation status was not assessed. ${ }^{10}$

Future studies are needed to evaluate the use of cisplatin in combination with other agents. Importantly, these studies should also address different individual patient sensitivity and de novo or acquired resistance to cisplatin revealed by the assays that reflect relative genetic instability. The results of current study allow suggesting that BRCA mutation status may be a prognostic and predictive biomarker for PDA and that BRCA-associated PDAC patients may benefit from the addition of platinum agents to standard therapy.

Our data have not definitively shown the tumor response for all stages and it seems reasonable to compare further the results in early stage vs late stage in a casecontrol study. A randomized clinical trial evaluating the addition to platinum-based therapy in a selected population of BRCA1, BRCA2 or PALB2 mutation carriers with PDA is currently ongoing.

In two patients who completed chemoradiotherapy, the post-treatment $\mathrm{CT}$ scans showed significant response to the treatment with complete lesion regression. After pancreatic resection, no residual invasive carcinoma was observed.

Post-treatment response in specimens has been shown to correlate with the prognosis in patients with several types of carcinomas including esophageal cancer, rectal cancer and pancreatic cancer. Patients who achieved pCR after neoadjuvant therapy had a better prognosis. Qing Zhao et al. ${ }^{21}$ have identified $2.5 \%$ of patients with pCR in a large cohort of 442 patients with PDAC who completed neoadjuvant CRT and subsequently underwent pancreatectomy. Patients with pCR had better overall survival than those with post-therapy stage I and stage IIA carrying residual invasive PDAC. ${ }^{21}$ It is also important to evaluate the prognostic significance of pathologic response to the neoadjuvant therapy in future studies.

In summary, surgical oncologists should consider BRCA mutation testing when the diagnosis of pancreatic cancer is established, especially when there is clinical or demographic reason to suspect a genetic predisposition. Recently, researches have demonstrated a survival advantages in patients with BRCA mutations and the above malignancies are strongly considered to be treated with the addition of a DNA cross-linking agent, such as cisplatin to a standard chemotherapy backbone. Future collaborative studies of a larger patient population are needed to confirm the prognostic value of $\mathrm{pCR}$ in patients with PDAC and evaluate the significance of the mutation locus in BRCA1/2 genes regarding the treatment outcomes in patients with BRCA-positive PDAC. The role of other factors influencing the grade and type of the response to therapy in above patients should be also assessed.

\section{REFERENCES}

1. Siegel RL, Miller KD, Jemal A. Cancer statistics, 2015. CA Cancer J Clin 2015;65:5-29.

2. Permuth-Wey J, Egan KM. Family history is a significant risk factor for pancreatic cancer: results from a systematic review and meta-analysis. Fam Cancer 2009;8:109-117.

3. Moran A, O'Hara C, Khan S, Shack L, Woodward E, Maher ER, et al. Risk of cancer other than breast or ovarian in individuals with BRCA1 and BRCA2 mutations. Fam Cancer 2012;11:235242.

4. Goggins M, Schutte M, Lu J, Moskaluk CA, Weinstein CL, Petersen GM, et al. Germline BRCA2 gene mutations in patients with apparently sporadic pancreatic carcinomas. Cancer Res 1996;56:5360-5364.

5. Iqbal J, Ragone A, Lubinski J, Lynch HT, Moller P, Ghadirian $\mathrm{P}$, et al. The incidence of pancreatic cancer in BRCA1 and BRCA2 mutation carriers. Br J Cancer 2012;107:2005-2009.

6. Matsubayashi H, Takaori K, Morizane C, Maguchi H, Mizuma M, Takahashi H, et al. Familial pancreatic cancer: concept, management and issues. World J Gastroenterol 2017;23:935-948.

7. Golan T, Kanji ZS, Epelbaum R, Devaud N, Dagan E, Holter $\mathrm{S}$, et al. Overall survival and clinical characteristics of pancreatic cancer in BRCA mutation carriers. Br J Cancer 2014;111:11321138 . 
8. Lowery MA, Kelsen DP, Stadler ZK, Yu KH, Janjigian YY, Ludwig E, et al. An emerging entity: pancreatic adenocarcinoma associated with a known BRCA mutation: clinical descriptors, treatment implications, and future directions. Oncologist 2011; 16:1397-1402.

9. Sonnenblick A, Kadouri L, Appelbaum L, Peretz T, Sagi M, Goldberg Y, et al. Complete remission, in BRCA2 mutation carrier with metastatic pancreatic adenocarcinoma, treated with cisplatin based therapy. Cancer Biol Ther 2011;12:165-168.

10. Fogelman D, Sugar EA, Oliver G, Shah N, Klein A, Alewine $\mathrm{C}$, et al. Family history as a marker of platinum sensitivity in pancreatic adenocarcinoma. Cancer Chemother Pharmacol 2015; 76:489-498.

11. Murphy KM, Brune KA, Griffin C, Sollenberger JE, Petersen GM, Bansal R, et al. Evaluation of candidate genes MAP2K4, MADH4, ACVR1B, and BRCA2 in familial pancreatic cancer: deleterious BRCA2 mutations in 17\%. Cancer Res 2002;62: 3789-3793.

12. Ryan DP, Hong TS, Bardeesy N. Pancreatic adenocarcinoma. N Engl J Med 2014;371:1039-1049.

13. Greer JB, Whitcomb DC. Role of BRCA1 and BRCA2 mutations in pancreatic cancer. Gut 2007;56:601-605.

14. Holter S, Borgida A, Dodd A, Grant R, Semotiuk K, Hedley D, et al. Germline BRCA mutations in a large clinic-based cohort of patients with pancreatic adenocarcinoma. J Clin Oncol 2015; 33:3124-3129.

15. Malone KE, Daling JR, Doody DR, Hsu L, Bernstein L, Coates $\mathrm{RJ}$, et al. Prevalence and predictors of BRCA1 and BRCA2 mutations in a population-based study of breast cancer in white and black American women ages 35 to 64 years. Cancer Res 2006; 66:8297-8308.
16. Tun NM, Villani G, Ong K, Yoe L, Bo ZM. Risk of having BRCA1 mutation in high-risk women with triple-negative breast cancer: a meta-analysis. Clin Genet 2014;85:43-48.

17. Couch FJ, Farid LM, DeShano ML, Tavtigian SV, Calzone K, Campeau L, et al. BRCA2 germline mutations in male breast cancer cases and breast cancer families. Nat Genet 1996;13: 123-125.

18. Alsop K, Fereday S, Meldrum C, deFazio A, Emmanuel C, George J, et al. BRCA mutation frequency and patterns of treatment response in BRCA mutation-positive women with ovarian cancer: a report from the Australian Ovarian Cancer Study Group. J Clin Oncol 2012;30:2654-2663.

19. Ielpo B, Caruso R, Duran H, Diaz E, Fabra I, Malavé L, et al. A comparative study of neoadjuvant treatment with gemcitabine plus nab-paclitaxel versus surgery first for pancreatic adenocarcinoma. Surg Oncol 2017;26:402-410.

20. Golan T, Sella T, O'Reilly EM, Katz MH, Epelbaum R, Kelsen $\mathrm{DP}$, et al. Overall survival and clinical characteristics of BRCA mutation carriers with stage I/II pancreatic cancer. Br J Cancer 2017;116:697-702.

21. Zhao Q, Rashid A, Gong Y, Katz MH, Lee JE, Wolf R, et al. Pathologic complete response to neoadjuvant therapy in patients with pancreatic ductal adenocarcinoma is associated with a better prognosis. Ann Diagn Pathol 2012;16:29-37.

22. Vyas O, Leung K, Ledbetter L, Kaley K, Rodriguez T, Garcon $\mathrm{MC}$, et al. Clinical outcomes in pancreatic adenocarcinoma associated with BRCA-2 mutation. Anticancer Drugs 2015;26:224-226.

23. Gostimir M, Bennett S, Moyana T, Sekhon H, Martel G. Complete pathological response following neoadjuvant FOLFIRINOX in borderline resectable pancreatic cancer - a case report and review. BMC Cancer 2016;16:786. 